\title{
A rare disease in childhood: angiokeratoma circumscriptum naviforme
}

\section{Mohammed Chaouche, Abdellah Dah Cherif, Selma El Kadiri, Sara Elloudi, Hanane Baybay, Fatima Zahra Mernissi}

\author{
Department of Dermatology and Venereology, University Hospital Hassan II, Fez, Morocco
}

Corresponding author: Dr. Mohammed Chaouche, E-mail: medch11@hotmail.com

\begin{abstract}
Angiokeratoma circumscriptum naviforme $(\mathrm{ACN})$ are sharply circumscribed hyperkeratotic vascular lesions that are often located on the lower extremities. It is called as naevoid (naviforme) since it is seen at birth or after birth and it is the rarest type of angiokeratomas. ACN is not related with other systemic diseases. Lesions of the disease are usually located in the extremities and are unilaterally localized. In this article, we present a case of seven-year-old girl with typical clinical manifestations.
\end{abstract}

Key words: Angiokeratoma circumscriptum naviforme; Birth; Bleeding

\section{INTRODUCTION}

Angiokeratomas are relatively rare vascular lesions, the precise mechanism for their development yet to be known. Variants of angiokeratomas include solitary and multiple angiokeratoma, angiokeratoma circumscriptum, angiokeratoma of Fordyce, angiokeratoma of Mibelli, and angiokeratoma corporis diffusum [1]. Angiokeratoma circumscriptum is the rarest variant. Clinically, it is manifested as dark red to blue-black nodules or plaques presenting unilaterally over the lower extremities. Other sites of presentation include thighs, buttocks, and so on. The lesions often become keratotic and show no tendency for spontaneous remission, posing a concern for cosmesis to the patient [2].

\section{CASE REPORT}

A 7-year-old girl was referred to us for a congenital lesion on her right axilla in a linear fashion. These lesion had gradually started to increase in size since last 6 years. She gave a history of occasional pain and several episodes of bleeding from the lesions even upon trivial trauma. There were no other systemic symptoms or history of external bleeding from any other site. Family history was noncontributory.

During dermatological examination, sharply circumscribed, about $5 \times 2 \mathrm{~cm}$ sized, dark red colored, hyperkeratotic vascular lesions in linear distribution on the right axilla were observed (Fig. 1). The dermoscopic patterns were characterized by a whitish veil covered with scales and red-dark lacunae (Fig. 2). ACN was diagnosed after histopathology and clinical examination. Chirurgical excision was performed with good outcome.

\section{DISCUSSION}

Angiokeratomas are relatively rare vascular lesions, with a collection of vessels, horn and tumor respectively refers to the presence of one or more dilated vessel(s) in the papillary dermis accompanied by an overlying acanthotic and/or hyperkeratotic epidermis. The worldwide prevalence is estimated to be about $0.16 \%$ with a female preponderance [3]. The pathogenesis is yet to be elucidated. According to the clinical

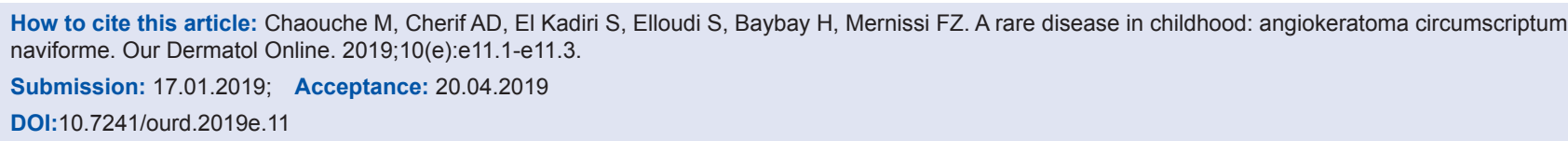




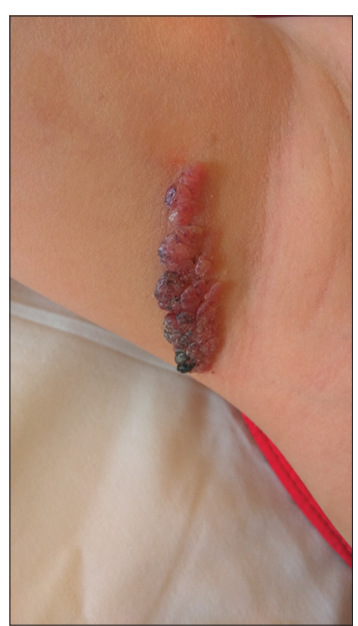

Figure 1: Angiokeratoma circumscriptum naviforme of axilla

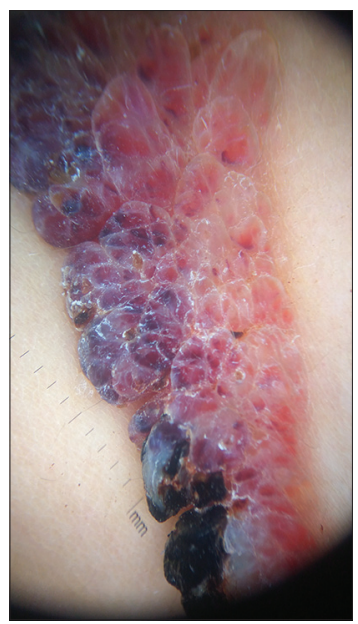

Figure 2: The dermoscopic patterns characterized by a whitish veil covered with scales and red-dark lacunae

appearance and evolution, there are five types of Angiokeratomas [4]: angiokeratoma of Mibelli in which cold temperature may precipitate acral lesions; angiokeratoma of Fordyce, the predominantly genital variety; angiokeratoma circumscriptum (AKC), which is nevoid with an early age of onset; solitary or multiple angiokeratoma with an onset in adult life; and angiokeratoma corporis diffusum (AndersonFabry disease), a systemic variety associated with widespread cutaneous lesions due to deposition of glycosphingolipids chiefly in the vascular endothelium and smooth muscle cells.; as a result of deficiency of the enzyme ceramide trihexosidase.

Angiokeratoma circumscriptum neviforme is the rarest variety, only a handful of cases having being reported in the world literature. Clinically, it is characterized by the presence of well-circumscribed, bluish black warty plaques, which may bleed even on trivial trauma (as in our case). The most common sites of predilection are the lower extremities, thighs and the gluteal region; in a linear or segmental distribution. However, several uncommon sites have been reported rarely, such as neck [5], arm, and trunk including elbow [6], penis [7]. Even tongue [8] and oral cavity [9] have not been spared.

The precise pathogenesis of angiokeratoma circumscriptum is unknown. However, it is known that it occurs due to telangiectasia of preexisting veins and capillaries, in the papillary dermis [1]; it's not an angioma as the name may suggest. Several causal factors such as congenital development, pregnancy, trauma, subcutaneous hematomas, and tissue asphyxia, have been hypothesized.

Angiokeratoma circumscriptum neviforme has been reported to co-exist with other vascular malformations, including angiokeratomas of fordyce [10], Cobb syndrome, Klippel-Trenaunay syndrome [1], nevus flammeus, cavernous hemangioma, and traumatic arteriovenous fistula [11].

Under the microscope, the presence of dilated blood vessels restricted to the papillary dermis along with overlying epidermal hyperkeratosis, papillomatosis, and/or acanthosis is a hallmark finding for the diagnosis of ACN. When the vascular proliferation extends deeper down involving the reticular dermis or subcutaneous fat, it hints toward the diagnosis of verrucous hemangioma, which may clinically resemble ACN.

As spontaneous regression is less likely, this condition demands definitive intervention. While diathermy, curettage, electrocautery, and cryosurgery are sufficient for smaller lesions, laser ablation (carbon dioxide [11], argon laser, potassium titanyl phosphate [KTP]) [4] or deep surgical excision are deemed suitable for larger lesions. Recently, KTP laser has been found to be superior, as it is very much specific for cutaneous blood vessels.

\section{CONCLUSION}

We have reported this uncommon case to hasten its prompt diagnosis in the coming days; although it may closely resemble some malignant skin tumors like malignant melanoma and pigmented basal cell carcinoma; thus relieving the patients of unnecessary psychological stress. Furthermore, an appropriate mode of treatment needs to be formulated, as this nevoid 
condition is persistent and liable to the episodes of bleeding and pain on trivial trauma.

\section{CONSENT}

The examination of the patient was conducted according to the Declaration of Helsinki principles.

\section{REFERENCES}

1. Mittal R, Aggarwal A, Srivastava G. Angiokeratoma circumscriptum: A case report and review of the literature. Int J Dermatol. 2005;44:1031-4.

2. Ghosh SK, Bandyopadhyay D, Ghoshal L, Haldar S. Angiokeratoma circumscriptum naeviforme: A case report of a rare disease. Dermatol Online J. 2011;17:11.

3. Schiller PI, Itin PH. Angiokeratomas: An update. Dermatology. 1996;193:275-82.

4. Sadana D, Sharma YK, Dash K, Chaudhari ND, Dharwadkar AA, Dogra BB. Angiokeratoma circumscriptum in a young male. Indian J Dermatol. 2014;59:85-7.

5. Sardana K, Koranne RV, Sharma RC, Mahajan S. Angiokeratoma circumscriptum naeviforme: Rare presentation on the neck. Australas J Dermatol. 2001;42:294-5.

6. Wang H, Zhang GY, Yu YQ. Variant distribution of skin lesions in angiokeratoma circumscriptum: Report of an untypical case. J Med Case.2012;3:297-9.

7. Saha M, Barlow R, Bunker CB. Angiokeratoma circumscriptum of the penis. Br J Dermatol. 2006;154:775-6.

8. Aggarwal K, Jain VK, Jangra S, Wadhera R. Angiokeratoma circumscriptum of the tongue. Indian Pediatr. 2012;49:316-8.

9. Kumar MV, Thappa DM, Shanmugam S, Ratnakar C. Angiokeratoma circumscriptum of the oral cavity. Acta Derm Venereol. 1998;78:472.

10. Feramisco JD, Fournier JB, Zedek DC, Venna SS. Eruptive angiokeratomas on the glans penis. Dermatol Online J. 2009;15:14.

11. del Pozo J, Fonseca E. Angiokeratoma circumscriptum naeviforme: Successful treatment with carbon-dioxide laser vaporization. Dermatol Surg. 2005;31:232-6.

Copyright by Mohammed Chaouche, et al. This is an open-access article distributed under the terms of the Creative Commons Attribution License, which permits unrestricted use, distribution, and reproduction in any medium, provided the original author and source are credited. Source of Support: Nil, Conflict of Interest: None declared. 